\title{
Systematic Review of Methodical Local Studies Re Usefulness of Antidepressants on Negative Symptoms of Schizophrenia
}

\author{
Saeed Shoja Shafti* \\ Full Professor of Psychiatry, University of Social Welfare and Rehabilitation Sciences (USWR), Iran
}

*Corresponding author: Saeed Shoja Shafti, Full Professor of Psychiatry, University of Social Welfare and Rehabilitation Sciences (USWR), Razi Psychiatric Hospital, Tehran, Iran.
Received Date: January 11, 2021

Published Date: February 02, 2021

\section{Abstract}

Introduction: Though negative symptoms are a real barricade against effective recovery in schizophrenia, their management by add-on antidepressants has produced changeable consequences. In the current study, some of the local systematic published studies have been the subject of a systematic review, to assess the effectiveness of adjunctive antidepressants on negative symptoms of schizophrenia.

Methods: Following probing in identified databank, eight home-based appropriate randomized clinical trials, containing 277 male participants, was nominated. All samples were selected among the long-lasting male patients, meeting the diagnosis of schizophrenia in line with 'Diagnostic and Statistical Manual of Mental Disorders', 4th edition, text revision, who entered into double-blind studies for random assignment to an antidepressant or placebo in addition to their current antipsychotic medications. In the said trials, the 'Scale for Assessment of Negative Symptoms' was the primary outcome measure for assessment of negative symptoms. Moreover, response was demarcated as a decrease of $\geqslant 20 \%$ in the score of the said measure (total and/or subscales).

Results: analysis of Combined Effect Size showed a significant positive influence of antidepressants on negative symptoms of schizophrenia. Similar results, too, were palpable separately with reference to different sub-scales of primary outcome measure. Anhedonia -Asociality displayed the best response, followed by Affective Blunting, Avolition -Apathy, Attention Deficit, and finally Alogia. Heterogeneity of the said analysis was insignificant and acceptable.

Conclusion: Antidepressants have promising sound effects with respect to improvement of negative symptoms of schizophrenia.

Keywords: Schizophrenia; Negative symptoms; Deficit syndrome; Adjuvant antidepressants; Noradrenergic antidepressants; Serotonergic antidepressants; Pharmacotherapy

\section{Introduction}

Schizophrenia is a heterogeneous syndrome characterized by positive, negative, and cognitive symptoms [1]. While positive symptoms refer to novel psychological experiences outside the range of normal (such as, hallucinations, delusions [2]. Schizophrenia is a heterogeneous syndrome characterized by positive, negative, and cognitive symptoms [1]. While positive symptoms refer to novel psychological experiences outside the range of normal [such as, hallucinations, delusions), negative symptoms signify loss of or dinary function. Negative symptoms include blunting of affect, poverty of speech and thought, apathy, anhedonia, reduced social drive, loss of motivation, lack of social interest, and inattention to social or cognitive input [3]. Attentiveness in the negative symptoms of schizophrenia has risen promptly over the last years, paralleling a growing interest in functional and clinical recovery [4]. Schizophrenia's negative symptoms have traditionally been regarded as treatment-resistant, and the major contributor to low function lev- 
els and debilitation in most patients with schizophrenia [5]. About $25 \%$ of patients with schizophrenia have a condition called the deficit syndrome, defined by severe and persistent negative symptoms. Resolution of positive symptoms, even in the early stages of schizophrenia, does not necessarily translate to functional recovery; figures suggest that full functional/social recovery occurs in less than $15 \%$ of people with schizophrenia, with negative symptoms playing a significant role [6]. A related complication is secondary negative symptoms - loss of expressiveness, interest, and social drive that results from social anxiety, fear of social stigma, depression, or the neurological side effects of antipsychotic drugs [1]. While negative symptoms are thoroughly related to the thinking deficits, the border between negative and cognitive symptoms is not sharp [7]. Patients with schizophrenia perform poorly on tests of mental fluency and flexibility, particularly word fluency and the capacity to sustain attention and shift its focus when needed [8]. While debate continues regarding the interrelationship and contribution of negative versus cognitive symptoms to functional impairment, it is clear that their impact is substantial [4]. Undoubtedly, the introduction of the antipsychotic medications in the 1950's heralded a novel epoch in the management of schizophrenia. About $70-80 \%$ of individuals with schizophrenia who take antipsychotic drug will find that their positive symptoms such as delusions and hallucinations are reduced and in some cases they may vanish totally [9]. But, while antipsychotic drugs are very effective against the positive symptoms, such an operative treatment for the negative symptoms unhappily remains beyond our knowledge [2]. Maybe it is so because the roots of the negative symptoms are more complex than the positive ones and ingrained in psychological factors as in chemical alterations within the brain cells [10]. In the 1980s the second generation antipsychotics were introduced and are now the drugs of first choice for treating psychosis [11]. In the beginning, it gave the impression that these new drugs were not only as effective as the first generation antipsychotic medications in treating the positive symptoms but they were possibly effective against negative symptoms. But, in fact it was not so. Despite inconsistency in outcomes of published researches, certainly if there is any beneficial effect from the atypical antipsychotics on negative symptoms it is far less striking than their effect on positive symptoms [12] Some practitioners have used antidepressants in the treatment of negative symptoms and have claimed that in addition to their effectiveness in combating the depression they may also increase the blood levels of the antipsychotic drugs and hence increase their effectiveness, and not only help with the depressive symptoms but also with the negative symptoms $[4,9]$. On the other hand, some scholars believe that none of the drugs now available is a specific treatment for schizophrenia. All of them are roughly equally good at suppressing psychotic symptoms and equally ineffective against negative symptoms [13]. So, pharmacotherapy of negative symptoms by add-on antidepressants is not without discrepancy. For example, while scholars like Möller [14], Silver [15], Bauer et al. [16],
Rummel et al. [17] and Helfer et al. [18] believe in usefulness of adjunctive antidepressants on negative symptoms, other researchers like Kissling et al. [19], Stahl et al. [20], Potvin et al. [21], Singh et al. [22], and Fusar-Poli et al. [23] see that an unpredictable, ambiguous or useless approach. Hence, in the current study and based on our review question (are antidepressants useful in the management of negative symptoms?), some of the local systematic published studies have been the subject of a new met-analysis, to assess over again the efficacy of add-on antidepressants on deficit syndrome of schizophrenia.

\section{Methods}

According to defined search strategy, publications in all languages were searched by means of internet from the following databases: The Cochrane Central Register of Controlled Trials, Cochrane Schizophrenia Group's Specialized Register, PubMed, MEDLINE, EMBASE, PsycINFO, LILACS, and ISI Web of Science. The selection criteria included all homemade, relevant randomized clinical trials (RCTs), comparing antidepressants with placebo, with regard to improvement of negative symptoms in schizophrenic patients with prominent negative symptoms. So, as the main result of the above-mentioned protocol, eight studies were selected, all of which were during the last fourteen years in Razi psychiatric hospital, and all of them were less than 13 weeks in duration [2430]. These studies included 277 participants, all of whom were men $(100 \%)$. The average age of participants was $38.83+/-13.61$ years (Tables 1 ).

In addition, in all the aforementioned studies the primary analysis was carried out according to the intention-to-treat, last observation carried forward (LOCF) approach. All the other open trials, non-methodical assessments, unpublished try-outs, or systematic assessments by medications other than antidepressants, which had been performed with respect to negative symptoms, were from the analysis. As a shared protocol, all the samples had been chosen among the chronic male inpatients, as accessible sample, meeting the diagnosis of schizophrenia according to Diagnostic and Statistical Manual of Mental Disorders, $4^{\text {th }}$ edition, text revision [31], who had been entered into parallel group, double-blind studies for random assignment to an antidepressant or placebo plus their current antipsychotic. Since the field of research was restricted to the chronic male section of the psychiatric hospital, so, all the samples were among chronic male schizophrenic patients. Razi psychiatric hospital, as the largest psychiatrist center in the Middle East, with 1375 beds, including 610 beds specified to chronic patients, was the field of research. The division between target group and control group was according to the registered number of the beds; even numbers into target group and odd numbers into placebo group. In addition, while the studies were carried out consistent with the 'Declaration of Helsinki and Ethical Principles for Medical Research Involving Human Subjects' [32], the patients were informed about the procedure, and a signed in- 
formed consent was received from those who were interested in participating in the study. The inclusion criterion for all the trials, in addition to the diagnosis of schizophrenia, was the duration of at least 2 years of illness. Cases with comorbidities such as major depressive disorder, mental retardation, neurological disorders, medical complications, severe aggressiveness, medical deafness or muteness were excluded from the study. In addition, cases with diagnosis of schizoaffective disorder or cases that had been prescribed a long-acting depot (during the last 6 months) or atypical antipsychotics, antidepressants or lithium were excluded. The Scale for Assessment of Negative Symptoms (SANS) [33] was the primary outcome measure in these experiments for assessment of negative symptoms. In addition, the Scale for Assessment of Positive Symptoms (SAPS) [34], Simpson Angus Scale (SAS) [35], Hamilton Rating Scale for Depression (HAM-D) [36], and Mini-Mental Status Examination (MMSE) [37], had been used for comparison of the intervening parameters in this study. High negative symptom scores ( $>55 \%$ of total SANS, $\geqslant 66$ ), low positive symptoms scores $(<55 \%$ of total SAPS, $\geqslant 96)$, and low extrapyramidal symptom scores $(<25 \%$ of total SAS, $\leqslant 10$ ) were at the basis of inclusion criteria. To exclude depression and cognitive disturbances that could be confused with negative symptoms, HAM-D and MMSE used, respectively. HAM-D $>10$ and MMSE $<25$ were identified as likely depression and cognitive disturbance and could lead to patient exclusion. All patients, after a washout period of 7-10 days, received haloperidol (5-10 mg/day), and after that were randomized to either placebo or antidepressant group, as add-on augmentative treatments. Since higher doses of antidepressants, could increase the hazard of intensification of psychosis, and the aim of the aforesaid assessments was evaluating the effectiveness of augmentative drug on merely the negative symptoms of schizophrenia, so the lower dosages were selected, incidentally. The tablets were prescribed while previously ins erted into empty and similar capsules, which were prepared in this regard, to make patients blind about the procedure. The evaluator (a clinical psychiatrist), and the staff, as well, were unaware (blind) about the abovementioned panel and the type of medications prescribed for each group. While all patients remained hospitalized throughout the experiment, they were evaluated at baseline and certain intervals through the assessments by the primary and ancillary outcome measures. Moreover, assessment of heterogeneity, publication bias analysis and grading quality of evidence (GRADE) [38], had been accomplished, for further clarification of meta-analysis.

\section{Statistical analysis}

Response, as a general rule, was defined as a reduction of $\geqslant 20 \%$ in the severity of SANS score (total and/or subscales). Odds Ratio (OR), Confidence Interval (CI), and combined effect size had been analyzed for each study and symptom, separately. Meta-DiSc Version 1.4 [39] and Meta-Essentials Version 1.0 [40] were the statistical software tools for analysis.

\section{Results}

The primary analysis, in all studies, carried out according to the intention-to-treat analysis. Meanwhile, there was not any significant missing data (with respect to outcome, summary, people, or characteristics) in the selected studies. According to the final results, while the heterogeneity of meta-analysis model was suitable $(<25 \%)$, the combined effect size of the abovementioned studies has shown a significant efficacy with respect to the effectiveness of antidepressants on negative symptoms of schizophrenia $(O R=7.00$, $C I=3.79-12.93, z=7.49, p<0.000$ ) (Table 2) (Figure 1).

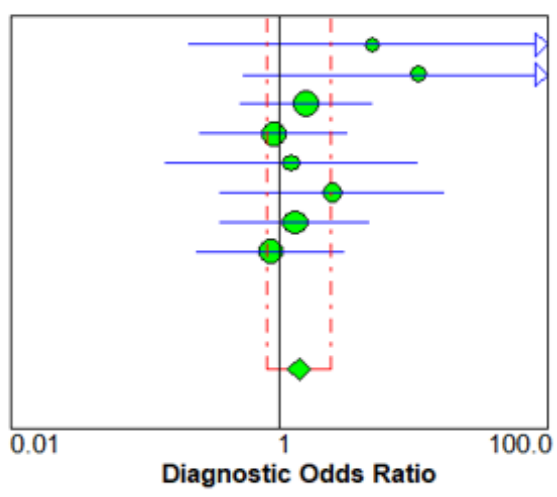

Citalopram

Clomipreamine

Fluoxetine

Nortriptyline

Maprotiline

Fluvoxamine

Reboxetine

Escitalopram

Random Effects Model

Pooled Diagnostic Odds Ratio $=1.40(0.81$ to 2.41$)$

Cochran- $Q=3.85 ;$ df $=7(p=0.7971)$

Inconsistency (I-square) $=0.0 \%$

Tau-squared $=0.0000$

Figure 1: Diagnostic Odds Ratios and pooled Odds Ratio (Random effects model).

Similar results also could be found regarding various subscales of SANS or symptoms of deficit syndrome, like Affective Blunting, Alogia, Avolition - Apathy, Anhedonia - Asociality, and Attention Deficit. According to the results, the Anhedonia -Asociality showed the best response $(O R=7.00, C I=3.79-12.39, z=7.49$, $p<0.000$ ), followed by Affective Blunting (OR=6.60, $C I=3.22-13.53$. $z=6.21, p<0.000)$, Avolition -Apathy $(O R=4.77, C I=2.38-9.57, z=$ 5.31, $p<0.000)$, Attention Deficit $(O R=3.74, C I=2.01-6.97, z=5.02$, $p<0.000$ ), and Alogia (OR=2.57, $C I=1.18-2.56, z=2.86, p<0.004)$, in sequence (Table 3). 
Table 1: Characteristics of involved studies.

\begin{tabular}{|c|c|c|c|c|}
\hline Target drug & Year of Study & Mode of Study & Technique of Evaluation & Number of Samples \\
\hline Citalopram $^{24}$ & 2003 & DB & RCT & 20 \\
\hline Clomipreamine $^{24}$ & 2003 & DB & RCT & 20 \\
\hline Fluoxetine $^{25}$ & 2004 & DB & RCT & 48 \\
\hline Nortriptyline $^{25,26}$ & 2004 & DB & RCT & 49 \\
\hline Maprotiline $^{27,28}$ & 2005 & DB & RCT & 20 \\
\hline Fluvoxamine $^{27,28}$ & 2005 & DB & RCT & 20 \\
\hline Reboxetine $^{29}$ & 2015 & DB & RCT & 50 \\
\hline Escitalopram $^{30}$ & 2007 & DB & RCT & 50 \\
\hline
\end{tabular}

Abbreviations: Double Blind=DB; Randomized Controlled Trial $=\mathrm{RCT}$

Table 2: Overall result of analysis, based on random effects model.

\begin{tabular}{|c|c|}
\hline \multicolumn{1}{|c|}{ Combined Effect Size } & 7 \\
\hline Odds Ratio & 3.79 \\
\hline CI Lower limit & 12.93 \\
\hline CI Upper limit & 3.79 \\
\hline PI Lower limit & 12.93 \\
\hline PI Upper limit & 7.49 \\
\hline Z-value & 0 \\
\hline One-tailed p-value & 0 \\
\hline Two-tailed p-value & 277 \\
\hline Number of incl. subjects & 8 \\
\hline Number of incl. studies & \\
\hline Neterogeneity of Meta-Analysis Model & 5.83 \\
\hline $\mathrm{p}_{Q}$ & 0.56 \\
\hline $\mathrm{I}^{2}$ & $0.00 \%$ \\
\hline T (Odds Ratio & 0 \\
\hline
\end{tabular}

Table 3: Positive response of various sub-scales of SANS (Pooled Outcome).

\begin{tabular}{|c|c|c|c|c|c|}
\hline Negative Symptoms & Odds Ratio & CI & Z-value & p-value & Heterogeneity $\left(\mathbf{I}^{2}\right)$ \\
\hline Anhedonia - Asociality & 7.00 & $3.79,12.93$ & 7.49 & 0.000 & $0.00 \%$ \\
\hline Affective Blunting & 6.60 & $3.22,13.53$ & 6.21 & 0.000 & $0.00 \%$ \\
\hline Avolition-Apathy & 4.77 & $2.38,9.57$ & 5.31 & 0.000 & $0.00 \%$ \\
\hline Attention Deficit & 3.74 & $2.01,6.97$ & 5.02 & 0.000 & $0.00 \%$ \\
\hline Alogia & 2.57 & $1.18,5.60$ & 2.86 & 0.004 & $15.53 \%$ \\
\hline
\end{tabular}

Heterogeneity of all the aforesaid subscales, as well, was minimal and consequently proper. Based on a domain-based evaluation, since no obvious bias (with respect to choice, performance, detection, attrition, and reporting) was clear, the risk of bias in this regard was generally low. Also, the publication bias analysis showed an adjusted combined effect size $=1.95$, in comparison with the observed combined effect size $=1.76$ (difference $=0.19$ ), which, while nominal, does not disdain the necessity for further methodical studies. Due to type of evidence (Randomized trials), no serious limitation to study quality, acceptable consistency, palpable directness, shortage of imprecise or sparse data ( except than three stud- ies with wide confidence intervals), and low chance of reporting bias, from one hand, and strong evidence of association with significant Odds Ratio of $>2$, based on consistent evidence from at least two observational studies, with no plausible confounders, Grading quality of evidence and strength of recommendations (GRADE) for this assessment sounds positive and satisfactory [38].

\section{Discussion}

Prevalent occurrence of negative symptoms and their resistance against available treatments, while they represent the most incapacitating and obstinate aspect of schizophrenia, make them 
tough to ignore. Consequently, interest in negative symptoms resurged in the 1980s-90s, with intense efforts to better comprehend them and manage them more efficiently [41].So, negative symptoms stay relevant because they are the main barrier to a better quality of life for patients with schizophrenia [2]. To this last point, work focusing on the prodromal phase of schizophrenia indicates that deficit symptoms and cognitive impairment are clear by the time of the first psychotic break [42], while enduring primary negative symptoms are identifiable in about $25-30 \%$ of people with chronic schizophrenia [43]. Generally, the four major clinical subgroups of negative symptoms are classifiable as affective, communicative, conational, and relational [44]. On the other hand, though deficit syndrome has a major impact on quality of life of the affected subject [45], they are often associated with a limited response to pharmacotherapy [46]. Thus far, studies specifically investigating negative symptoms in schizophrenia are scant [47]. Therefore, proper selection of therapy of negative symptoms remains a major unmet medical need [48], and, according to the heterogeneity of the clinical presentation of schizophrenia, treatment of negative symptoms, as well as therapy for other associated symptoms, must be largely individualized according to a patient's specific characteristics [49]. Though since the 1950s the introduction of effective pharmacological treatments for positive symptoms has slowly shifted the diagnostic process toward a positive symptom-based model [50], during the 1980s, a number of classifications of schizophrenia based on negative symptoms were offered [51], which revealed, nevertheless, several restrictions, including the lack of diagnostic stability over time and the poor prognostic value [52]. Back to our discussion and according to the finding of the present study, antidepressants have significant effectiveness for amelioration of negative symptoms. In this regard, the greatest effect was evident with respect to anhedonia - asociality and the slightest effect had been demonstrated with regard to alogia. So, our conclusions are in complete harmony with Möller [14], Silver [15], and Bauer et al. [16], who had found antidepressant medications useful for amelioration of negative symptoms. Also, it is rather compatible with the findings of Kissling et al. [19], who had found combination of antipsychotics and antidepressants effective in treating negative symptoms of schizophrenia, though as said by him the amount of information was too limited to allow any firm conclusions. In this regard, while they found no significant difference for 'leaving the study early due to adverse events' and 'leaving the study early due to inefficacy' between the two treatment groups, participants treated with the antipsychotic plus antidepressant medications showed meaningfully greater improvement in negative symptoms than those treated with antipsychotics alone. Also, significant difference in favor of the combination therapy was observable in different features of negative symptoms, like 'affective flattening', 'alogia' and 'avolition'; which was again comparable to the results of the present assessment. Similarly, our conclusions were coherent with the outcomes of Rummel et al. [17] and Helfer et al. [18], who found that antidepressants were effective in reducing negative symptoms, with a medium to high effect size, and such a reduction may be at least partly independent from the antidepressant effect. In contrast, our conclusions are not compatible with the findings of Stahl et al. [20], who had stated that the 'measurement and treatment trials' methodology for the evaluation of negative symptoms need more refinement before therapeutic optimism that better treatments for negative symptoms can be recognized. Also, in contrary to our findings, which included both serotonin-specific reuptake inhibitors (SSRIs) and tricyclic antidepressants, it is not in agreement with Potvin et al. [21], who did not find any significant efficacy as regards the SSRIs. Nonetheless, when their studies were divided according to severity of illness, a moderate and significant effect size emerged for the studies involving the so-called 'chronic patients'. Anyhow, according to their final conclusion, the completed meta-analysis could not deliver any inclusive support for the SSRIs. Maybe, according to Czobor et al., while general efficacy outcomes seem to be positive, proof for effectiveness of current psychopharmacological medications is difficult to assess because of the procedural problems and changeable consequences [53]. Also, the present analysis is not in agreement with the results of Hinkelmann et al. [54], who did not find any differences between serotonergic versus noradrenergic antidepressants, as add-on therapy for negative symptoms.

Likewise, our results were not consistent with the conclusions of Singh et al. [24], and Fusar-Poli et al. [25], who believed that despite generally reporting an effective response to antidepressive agents in treating negative symptoms with small to medium effect sizes, such studies often did not use a stringent definition of negative symptoms (eg, prominent/predominant negative symptoms) or control for potential antidepressant effects. Thus, effects on secondary negative symptoms, again, could not be ruled out. For sure, efforts continue to better define and measure negative symptoms, distinguish their impact from that of other symptom domains, and establish effective managements as well as appraisals to assess them [44]. For example, by studying endophenotypes, scientists hope to learn how these processes work - not only in persons with schizophrenia, but in other people who may or may not have a schizophrenia spectrum disorder. As research links subtle signs and symptoms of disease to a person's basic genetic makeup, we will probably better comprehend this range of illnesses, a process which will make it easier to develop specific treatments, along with better ways for clinicians to select a useful treatment [55]. Nonetheless, the mechanism by which adding an antidepressant to antipsychotic medication may cause a reduction in negative symptoms is still unclear. If a non-specific antidepressant effect cannot be excluded, it is also possible that the antidepressants' effectiveness may be influenced by the pharmacological action of the concurrent antipsychotic drug [45]. More recently, the National Institute of Mental Health (NIMH) Measurement and Treatment Research to Improve Cognition in Schizophrenia (MATRICS) turned its attention to negative symptoms. From a treatment perspective, it is noteworthy that the MATRICS consensus statement makes refer- 
ence to persistent negative symptoms and indicates the distinction between primary and secondary negative symptoms is not essential [7], which is many times unattainable [56-59]. No doubt, while minimal heterogeneity, identical sampling and similar chronicity of the cases were among the strong points of the current evaluation, limited number of methodical local studies, gender-based sampling, short-term period of assessments, and miscellaneous sampling sizes were among the weaknesses of the present study, which together may limit the generalizability of findings.

\section{Conclusion}

Antidepressants have promising sound effects with respect to improvement of negative symptoms of schizophrenia.

\section{Acknowledgement}

None.

\section{Conflict of Interest}

No conflict of interest.

\section{References}

1. Carpenter WT, Heinrichs DW, Wagman AMI (1988) Deficit and nondeficit forms of schizophrenia: the concept. Am J Psychiatry 145: 578-583.

2. Tandon R, Jibson M (2002) Negative symptoms of schizophrenia: How to treat them most effectively. Current Psychiatry 1(9): 36-42.

3. Kirkpatrick B, Buchanan RW, McKenney PD, Alphs LD, Carpenter WT (1989) The Schedule for the Deficit Syndrome: an instrument for research in schizophrenia. Psychiatry Res 30(2): 119-124.

4. Remington G, Foussias G, Fervaha G, Agid O, Takeuchi H, et al. (2016) Treating Negative Symptoms in Schizophrenia: an Update. Curr Treat Options Psych 3: 133-150.

5. Andreasen NC (1982) Negative symptoms in schizophrenia: definition and reliability. Arch Gen Psychiatry 39: 784-788.

6. Austin SF, Mors O, Secher RG, Hjorthoj CR, Albert N, et al. (2013) Predictors of recovery in first episode psychosis: the opus cohort at 10year follow-up. Schizophr Res 150(1):163-168.

7. Kirkpatrick B, Fenton WS, Carpenter WT Jr, et al. (2006) The NIMHMATRICS consensus statement on negative symptoms. Schizophr Bull 32(2): 214-219.

8. Barch DM, et al. (2005) The Relationships among Cognition, Motivation, and Emotion in Schizophrenia: How Much and How Little We Know. Schizophrenia Bulletin 31(4): 875-81.

9. Meltzer HY, Sommers AA, Luchins DJ (1986) The effect of neuroleptics and other psychotropic drugs on negative symptoms in schizophrenia. J Clin Psychopharmacol 6: 329-338.

10. Tandon R, Greden JF (1989) Cholinergic hyperactivity and negative schizophrenic symptoms. Arch Gen Psychiatry 46: 745-753.

11. Breier A, Buchanan RW, Kirkpatrick B, et al. (1994) Effect of clozapine on positive and negative symptoms in outpatients with schizophrenia. Am J Psychiatry 151(1): 20-26.

12. DeQuardo JR, Tandon R (1998) Do atypical antipsychotic medications favorably alter the long-term course of schizophrenia? J Psychiatric Res 32: $229-242$

13. Hunter R, Barry S (2012) Negative symptoms and psychosocial functioning in schizophrenia: neglected but important targets for treatment. Eur Psychiatry 27(6): 432-436.

14. Möller HJ (2003) Management of the negative symptoms of schizophrenia: new treatment options CNS Drugs 17(11): 793-823.
15. Silver H (2004) Selective serotonin re-uptake inhibitor augmentation in the treatment of negative symptoms of schizophrenia. Expert Opin Pharmacother 5(10): 2053-8.

16. Bauer A, Godemann F, Reischies FM, Selig F, Schlattmann P, et al. (2005) Negative symptoms of schizophrenia are improved by the addition of paroxetine to neuroleptics: a double-blind placebo-controlled study. Int Clin Psychopharmacol 20(1): 27-31.

17. Rummel C, Kissling W, Leucht S (2005) Antidepressants as addon treatment to antipsychotics for people with schizophrenia and pronounced negative symptoms: a systematic review of randomized trials. Schizophr Res 80 (1): 85-97.

18. Helfer B, Samara MT, Huhn M, Elisabeth Klupp, Claudia Leucht, et al. (2016) Efficacy and safety of antidepressants added to antipsychotics for schizophrenia: a systematic review and meta-analysis. Am J Psychiatry 173(9): 876-886.

19. Kissling W, Leucht S Rummel C (2006) Antidepressants for the negative symptoms of schizophrenia. Cochrane Database Syst Rev (3): CD005581.

20. Stahl SM, Buckley PF (2007) Pharmacological treatment of negative symptoms of schizophrenia: therapeutic opportunity or cul-de-sac? Acta Psychiatr Scand 115(2): 93-100.

21. Potvin S, Elie R, Stip E, Sepehry AA (2007) Selective serotonin reuptake inhibitor (SSRI) add-on therapy for the negative symptoms of schizophrenia: a meta-analysis. J Clin Psychiatry 68(4): 604-10.

22. Singh SP, Singh V, Kar N, Chan K (2010) Efficacy of antidepressants in treating the negative symptoms of chronic schizophrenia: meta-analysis. Br J Psychiatry 197(3): 174-179.

23. Fusar-Poli P, Papanastasiou E, Stahl D, et al. (2015) Treatments of negative symptoms in schizophrenia: meta-analysis of 168 randomized placebo-controlled trials. Schizophr Bull 41(4): 892-899.

24. Shoja Shafti (2003) S: Effectiveness of Citalopram, Alprazolam and Clomipramine in amelioration of negative symptoms in Schizophrenia. Journal of Rehabilitation 3(12): 42-49.

25. Shoja Shafti. S (2004) Effectiveness of Bromocriptine, Flouxetine and Nortriptyline in ameliorating the negative symptoms of Schizophrenia. Journal of Rehabilitation 5(16): 58-63.

26. Shoja Shafti S (2006) Treatment-responsiveness of negative symptoms in schizophrenia: a double-blind placebo-control clinical trial. Iranian J Med Sci 31: 135-138.

27. Shoja Shafti S (2004) Rehabilitation of Schizophrenia: Adjunctive Therapy of Negative Symptoms, Iranian Rehabilitation Journal 1(2):2231.

28. Shoja Shafti S (2005) Drug -Specific Responsiveness of Negative Symptoms. International Journal of Psychosocial Rehabilitation 10(1): 177-186.

29. Shoja Shafti S, Jafarabad MS, Azizi R (2015) Amelioration of deficit syndrome of schizophrenia by norepinephrine reuptake inhibitor. Therapeutic Advances in Psychopharmacology 1-8.

30. Shoja Shafti S (2007) Amelioration of negative symptoms in schizophrenia by escitalopram: a double blind, placebo-controlled clinical trial. Clin Schizophrenia Related Psychosis 1: 255-258.

31. American Psychiatric Association (APA) (2000) Diagnostic and Statistical Manual of Mental Disorders. 4th ed. Text rev. Washington, DC: American Psychiatric Association.

32. World Medical Association (2013) World Medical Association Declaration of Helsinki: ethical principles for medical research involving human subjects. JAMA 310(20): 2191-4.

33. Andreasen N (1981) The Scale for Assessment of Negative Symptoms (SANS), University of Iowa , Department of Psychiatry , Iowa City, Iowa.

34. Andreasen N (1984) The Scale for Assessment of Positive Symptoms (SAPS), University of Iowa , Department of Psychiatry , Iowa City, Iowa. 
35. Simpson GM, Angus JW (1970) A Rating Scale for Extrapyramidal Side Effects, Acta Psychiatr Scand; 212 (suppl 44):11-9.

36. Hamilton M (1960) Rating scale for depression. J Neurol Neurosurg Psychiatry 23: 56.

37. Folstein M F, Folstien S E, McHugh P R (1975) Mini- mental state." A practical method for grading the cognitive state of patients for the clinician. J Psychiatr Res 12: 189.

38. GRADE Working Group (2004) Grading quality of evidence and strength of recommendations. BMJ 328: 1 of 8.

39. Zamora J, Abraira V, Muriel A, Khan KS, Coomarasamy A (2006) MetaDiSc: a software for meta-analysis of test accuracy data. BMC Medical Research Methodology 6:31.

40. Van Rhee HJ, Suurmond R, Hak T (2015) User manual for MetaEssentials: Workbooks for meta-analysis (Version 1.0) Rotterdam, The Netherlands: Erasmus Research Institute of Management.

41. Carpenter WT, Heinrichs DW, Alphs LD (1985) Treatment of negative symptoms. Schizophrenia Bull 11: 440-452.

42. Bora E, Lin A,Wood SJ, Yung AR, McGorry PD, Pantelis C (2014) Cognitive deficits in youth with familial and clinical high risk to psychosis: a systematic review and metaanalysis. Acta Psychiatr Scand 130(1):1-15

43. Kirkpatrick B, BuchananRW, Ross DE, Carpenter Jr WT (2001) A separate disease within the syndrome of schizophrenia. Arch Gen Psychiatry 58(2): 165-171.

44. Cerveri G, Gesi C, Mencacci C (2019) Pharmacological treatment of negative symptoms in schizophrenia: update and proposal of a clinical algorithm. Neuropsychiatric Disease and Treatment 15: 1525-1535.

45. Blanchard JJ, Kring AM, Horan WP, et al. (2011) Toward the next generation of negative symptom assessments: the collaboration to advance negative symptom assessment in schizophrenia. Schizophr Bull 37(2): 291-299.

46. Leucht S, Davis JM (2017) Schizophrenia, primary negative symptoms, and soft outcomes in psychiatry. Lancet 389(10074): 1077-1078.

47. Németh G, Laszlovszky I, Czobor P, Erzsébet Szalai, Balázs Szatmári, et al. (2017) Cariprazine versus risperidone monotherapy for treatment of predominant negative symptoms in patients with schizophrenia: a randomised, double-blind, controlled trial. Lancet 389(10074): 11031113.

48. Corponi F, Serretti A, Montgomery S, Fabbri C (2017) Cariprazine specificity profile in the treatment of acute schizophrenia: a metaanalysis and meta-regression of randomized-controlled trials. Int Clin Psychopharmacol 32(6): 309-318.

49. Malaspina D, Walsh-Messinger J, Gaebel W, et al. (2014) Negative symptoms, past and present: a historical perspective and moving to DSM-5. Eur Neuropsychopharmacol 24(5): 710-724.

50. Crow TJ (1980) Molecular pathology of schizophrenia: more than one disease process? Bmj 280(7): 66-68.

51. Andreasen NC, Olsen S (1982) Negative v positive schizophrenia Definition and validation. Arch Gen Psychiatry 39: 789-794.

52. Peralta V, de Leon J, Cuesta MJ (1992) Are there more than two syndromes in schizophrenia? A critique of the positive-negative dichotomy. Br J Psychiatry 161: 335-343.

53. Czobor P, Möller HJ (2015) Pharmacological treatment of negative symptoms in schizophrenia. Eur Arch Psychiatry Clin Neurosci 265(7):567-78.

54. Hinkelmann K, Yassouridis A, Kellner M, Jahn H, Wiedemann K, et al. (2013) No effects of antidepressants on negative symptoms in schizophrenia. J Clin Psychopharmacol 33(5): 686-90.

55. Cannon TD, Matthew CK (2006) Endophenotypes in the Genetic Analyses of Mental Disorders. Annual Review of Clinical Psychology 2: 267-290.

56. Shoja Shafti S. Odyssey of 'Negative Symptoms' of Schizophrenia: Rehabilitation vs Stigmatization. Current Psychopharmacology 2015; 4(1): 1-12.

57. Shoja Shafti S. Primary Negative Symptoms: Appraisal of a Misty Outlook. American Journal of Biomedical Science \& Research 2019: 317-319.

58. Shoja Shafti S. Deficit Syndrome of Schizophrenia: Specific Serotonin Reuptake Inhibitors Set Against Tricyclic Antidepressants. International Journal of Psychiatry Research 2019; 1 (1): 12-14.

59. Shoja Shafti S. Evaluation of Assemblage and Manageability of Negative Symptoms of Schizophrenia: A New Stance. Journal of Psychiatry Studies 2019; 2 (1): 1-6. 